\title{
Signal performance analysis of the latest quartet of Galileo satellites during the first operational year
}

\author{
Constantin-Octavian Andrei*, Jan Johansson ${ }^{\dagger}$ Hannu Koivula*, Markku Poutanen*, \\ *Finnish Geospatial Research Institute, National Land Survey of Finland \\ $\dagger$ Chalmers University of Technology, Sweden \\ Email: firstname.lastname@\{nls.fi;chalmers.se\}
}

\begin{abstract}
On 12 February 2020, the latest four Galileo satellites had completed one full year of space operational service. The satellites were launched on 25 July 2018. The quartet increased the operational capacity of the Galileo constellation to 22 satellites. This study reports on three signal-in-space ( $\mathrm{SiS}$ ) performance indicators - status, availability, and ranging accuracy - from 11 February 2019 to 12 February 2020. In addition, the study looks also at how the on-board satellite clocks have performed. The data analysis shows $100 \%$ data validity and signal health status for three our of the four satellites, whereas NAPA (No Accuracy Prediction Available) events accounted for about 2\% of the time. In addition, SiS availability was higher than $95 \%$ in the first operational year. Furthermore, the 95th percentile of the global average of the instantaneous signal in space error is found to vary between 0.17 to $0.33 \mathrm{~m}$ on monthly basis. Lastly, the precise satellite clock biases show high short-term performance with $0.1 \mathrm{ps} / \mathrm{s}\left(10^{-13} \mathrm{~s} / \mathrm{s}\right)$ standard deviations during the first operational year. The numerical results indicate a robust performance and high reliability for the youngest Galileo satellites in the constellation. They increase the number of operational satellites in the constellation and thus contribute to the Galileo's increased popularity in the satellite-based positioning and navigation user community.
\end{abstract}

\section{Index Terms}

Galileo, SISE, SISA, DVS, SHS, performance, signal, health, validity, status, availability, accuracy, clock

\section{INTRODUCTION}

On 12 February 2020, the latest four Galileo satellite had completed one full year of operational service. GSAT0219, 0220, 0221 and 0222 were launched on 25 July 2018 and injected on four slots 


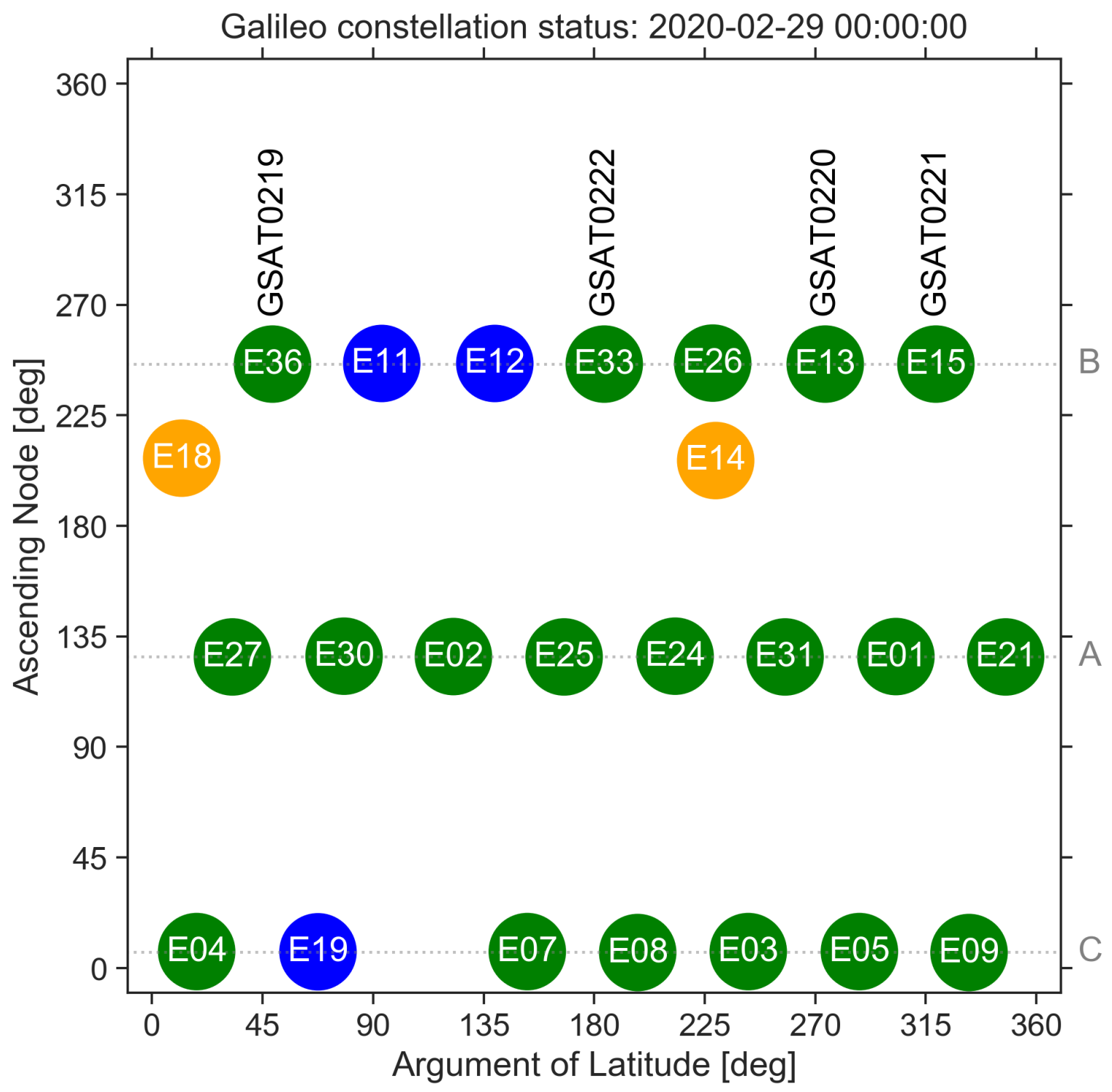

Fig. 1. Galileo constellation

of the orbital plane B of the Galileo constellation (Fig.1). The four satellites were marked ready for operation on 11 February 2019 around mid-day UTC time (see Table I). They raised to 22 the number of the operational satellites in the constellation. The quartet is special to the GNSS Nordic community as two of the satellites (GSAT0221 and GSAT0222) are nicknamed Anna and Ellen, after the winners of the Galileo children's drawing competition in Finland and Sweden, respectively.

Galileo has been offering initial services since mid December 2016. These services differ in the level of accessibility: free (Open Service, OS), encrypted (Public Regulated Service, PRS), and authenticated 
TABLE I

GALILEO SATELLITES LAUNCHED ON 25 JULY 2018.

\begin{tabular}{lcclr}
\hline SVID & $\begin{array}{c}\text { PRN } \\
\text { code }\end{array}$ & $\begin{array}{c}\text { Orbital } \\
\text { slot }\end{array}$ & Nickname & $\begin{array}{r}\text { Activation } \\
\text { 11-Feb-2019 }\end{array}$ \\
\hline GSAT0219 & E36 & B04 & Tara (SVK) & 10:26:00 UTC \\
GSAT0220 & E13 & B01 & Samuel (SLO) & 10:56:00 UTC \\
GSAT0221 & E15 & B02 & Anna (FIN) & 11:26:00 UTC \\
GSAT0222 & E33 & B07 & Ellen (SWE) & 12:10:00 UTC \\
\hline
\end{tabular}

(High Accuracy Service, HAS). In addition, Galileo satellites contribute to the search-and-rescue as well as safety-of-life global systems. The activation of the initial services led to an increase in number of publications reporting on the Galileo performance in terms of ranging, positioning and timing. [1] were among the first to report that the quality of broadcast orbits and clocks has improved since the beginning of routine transmission. Using a constellation of 12 active satellites, the researchers reported a signalin-space range error (SISE) of $30 \mathrm{~cm}$ and Galileo-only point positioning precision of $2 \mathrm{~cm}$ in static mode using daily solutions. [2] reported extensively on the Galileo status, methodology and performance metrics for the 2014-2017 time period. The authors also concluded that the global average SISE has improved gradually and stabilised around a constellation value of $0.50 \mathrm{~m}(95 \%)$ in 2017. In addition, the per-satellite availability was found to be above $87 \%$, whereas $100 \%$ availability was reported for the initial open services. In terms of timing performance, the UTC dissemination error was found to be $8.9 \mathrm{~ns}$ (95th percentile), whereas the GPS to Galileo offset (GGTO) dissemination accuracy was estimated to be $7.2 \mathrm{~ns}$ (95th percentile). Furthermore, the accuracy of the on-board clock was estimated to be $0.45 \mathrm{~cm}(95 \%)$ in September 2017. [3] reported global average root-mean-square signal-in-space range error (SISRE) values at the $0.20 \mathrm{~m}$ level and $95 \%$ percentile values of $0.30-0.50 \mathrm{~m}$ for different single- and dual-frequency services. They used data for the entire 2017 year and precise products from GeoForschungsZentrum Potsdam (GFZ) as reference. [4] confirmed the above results and extend the analysis by including all data in 2018. The authors found the average root mean square of SISRE for Galileo constellation to be $0.22 \mathrm{~m}$ in 2018, whereas the individual annual mean RMS for the operational satellites varied between 0.17 to $0.29 \mathrm{~m}$. The clock errors of Galileo satellites were found to be approximately $0.17 \mathrm{~m}$. Lastly, the European GNSS Agency has published the Open Service quarterly performance reports since 2017. The latest available report at the time of writing is Q3/2019.

To the best of authors' knowledge, this contribution is the first study to include or refer up to date 
TABLE II

GALILEO FREQUENCY BANDS, RANGING SIGNALS AND COMPONENTS

\begin{tabular}{lllllll}
\hline $\begin{array}{l}\text { Galileo } \\
\text { signal }\end{array}$ & $\begin{array}{l}\text { Carrier } \\
(\mathrm{MHz})\end{array}$ & $\begin{array}{l}\text { Sub } \\
\text { band }\end{array}$ & $\begin{array}{l}\text { Carrier } \\
(\mathrm{MHz})\end{array}$ & Message & Service & Components \\
\hline E1 & \multirow{2}{*}{1575.420} & & & I/NAV & OS & E1-B/C \\
& & & & & PRS & E1-A \\
E6 & \multirow{2}{*}{1278.750} & & & C/NAV & PRS & E6-A \\
& & & & & HAS & E6-B/C \\
E5 & \multirow{2}{*}{1191.795} & E5a & 1207.14 & F/NAV & OS & E5a-I/Q \\
& & E5b & 1176.45 & I/NAV & OS & E5b-I/Q \\
\hline
\end{tabular}

to the latest four Galileo satellites. Therefore, this study contributes with new information by focusing on the open signals transmitted by the latest quartet of the Galileo satellites. The study is structured as follows. Section I gives a brief introduction on the context this study was derived. Section II presents the methodology along with the definition of the performance indicators and data used in the study. Section III reports and discusses the findings based on the numerical analysis using data logged from the latest four Galileo satellites over the first operational year, i.e. 12 February 2019 to 12 February 2020. Section IV summarises the key points of this study together with the direction of the future work.

\section{Methodology}

Galileo open service is defined in three main documents describing the service use [5], the processing of the open signals [6], and the Galileo ionospheric model for single-frequency users [7]. These documents state that Galileo is a multi-frequency passive system that transmits different ranging signals and navigation messages. In addition, the documents define several key performance indicators and the minimum performance levels of the Galileo open services. For full details, the reader is pointed out to the relevant documents. Below, we give a brief summary on the aspects relevant for this study.

\section{A. Galileo Ranging Signals}

Galileo satellites transmit three independent ranging signals, named E5, E6 and E1. The E5 signal is further sub-divided into two signals denoted E5a and E5b. The open and commercial signals are provided as pairs of data and pilot components. All Galileo signals are derived from the same on-board master clock [8] and are transmitted on the L-band within 1.1 to $1.6 \mathrm{GHz}$. Table II summarises the Galileo frequency bands, ranging signals and components. 
The open service provides five Galileo signal combinations: three single-frequency signals (E1, E5a, E5b) and two dual-frequency signal combinations (E1/E5a, E1/E5b). The latter are valid under the assumption that the signals used in the combination come from the same satellite.

\section{B. Galileo Navigation Messages}

Table II shows that apart of the ranging signals, Galileo satellites are designed to also transmit three different types of navigation messages:

- F/NAV (Free NAVigation) message transmitted at low data rate,

- I/NAV (Integrity NAVigation) message transmitted at high data rate,

- C/NAV (Commercial channel NAVigation) message.

F/NAV message is solely used by the open service, whereas I/NAV messages corresponds to both open and commercial services. C/NAV message format has not been published yet. Thus, it is not part of the study. Both F/NAV and I/NAV provide direct support to position/velocity/time determination [8], through provision of several parameters specific to the service (time, data validity and signal health flags, ionosphere model, etc.) and transmitting satellite (ephemeris and clock corrections). Most of the parameters of the two messages are identical. However, the messages are optimised for a specific pair of frequencies. F/NAV is optimised for E1/E5a dual-frequency combination, whereas I/NAV is designed for E1/E5b dual-frequency combination. Therefore, the clock correction and broadcast group delay parameters are different and specific for F/NAV and I/NAV, respectively.

\section{Galileo Signal Performance Characteristics}

Reference [5] defines a number of key performance indicators based on several signal characteristics: status, availability and accuracy. We give below the definition of the indicators used in this study.

1) Signal status: The operational status of the signals transmitted by the Galileo satellites is embedded in the navigation message through three status flags: signal health status (SHS), data validity status (DVS) and signal-in-space accuracy (SISA). These flags determine three possible values for the status: healthy, marginal and unhealthy. Chapter 4 in [6] gives full details on these flags and their position within the navigation message. Section 2.3.1 in [5] provides guidelines on the mapping of all status flags into the signal-in-space status values. Table III shows which status flags must be checked when single-frequency and/or dual-frequency signals are to be used.

2) Per-satellite signal availability: Per-satellite signal availability is defined as the amount of time that a specific satellite transmits a healthy signal. The availability is expressed in percentage of time and includes planned and unplanned outages. 
TABLE III

GALILEO STATUS FLAGS CHECKED FOR DIFFERENT SIGNAL COMBINATION

\begin{tabular}{|c|c|c|c|c|c|c|}
\hline \multicolumn{2}{|c|}{ Navigation message } & \multicolumn{5}{|c|}{ Galileo Open Service Signal-in-Space } \\
\hline \multirow[t]{2}{*}{ type } & \multirow[t]{2}{*}{ flag } & \multicolumn{3}{|c|}{ Single-frequency } & \multicolumn{2}{|c|}{ Dual-Frequency } \\
\hline & & E1 & E5a & E5b & E1/E5a & $\mathrm{E} 1 / \mathrm{E} 5 \mathrm{~b}$ \\
\hline \multirow[t]{3}{*}{ F/NAV } & E5aSHS & & $\mathrm{x}$ & & $\mathrm{x}$ & \\
\hline & E5aDVS & & $\mathrm{x}$ & & $\mathrm{x}$ & \\
\hline & SISAE1E5a & & $\mathrm{x}$ & & $\mathrm{x}$ & \\
\hline \multirow[t]{5}{*}{ I/NAV } & E1BSHS & $\mathrm{x}$ & & & $\mathrm{x}$ & $\mathrm{x}$ \\
\hline & E1BDVS & $\mathrm{x}$ & & & $\mathrm{x}$ & $\mathrm{x}$ \\
\hline & E5bSHS & & & $\mathrm{x}$ & & $\mathrm{x}$ \\
\hline & E5bDVS & & & $\mathrm{x}$ & & $\mathrm{x}$ \\
\hline & SISAE1E5b & $\mathrm{x}$ & & $\mathrm{x}$ & & $\mathrm{x}$ \\
\hline
\end{tabular}

Please notice that the typical refresh rate of the navigation message transmitted by the Galileo satellites varies from 10 to 180 minutes. Furthermore, the maximum period of a healthy navigation message is set to 240 minutes since the ephemeris reference time that it is included in each navigation message [5]. After that, the data transmitted in the navigation message is considered expired and should not be used in the Galileo services.

3) Ranging accuracy: Galileo ranging accuracy is defined as the 95th percentile of the time series of the global average Galileo Signal-in-Space Error (SISE). The accuracy is measured only for the time periods during which the satellite transmitted a healthy signal.

SISE provides the instantaneous difference between the broadcast and reference Galileo satellite position and clock corrections, projected on the user-satellite direction. The broadcast values are obtained from the navigation message, whereas the reference values are taken, for example, from the IGS (International GNSS Service) final product. The instantaneous global average SISE can be estimated analytically from the orbit error components (along-track, cross-track and radial) and its total clock prediction error. Section C.4.3.2 in [5] gives the following approximation formula:

$$
\begin{aligned}
\mathrm{SISE}=( & 0.96910 \cdot R^{2}+C L K^{2} \\
& +0.01545 \cdot\left(A^{2}+C^{2}\right) \\
& +1.96881 \cdot C L K \cdot R)^{\frac{1}{2}}
\end{aligned}
$$


The coefficients provided in Eq. 1 come under the assumption that an elevation masking angle of 5 degrees is applied by the Galileo receivers. In addition, the following conventions are also considered:

- the radial direction $(\mathrm{R})$ is defined from the satellite towards the Earth centre.

- the cross direction (C) is the direction perpendicular to the orbital plane aligned with the cross product of the radius and velocity.

- the along direction (A) is perpendicular to the radius in the orbital plane and positive direction is in the same semi-plane of the velocity.

The statistics of the global average SISE are computed at 5-min intervals to match the precise products used as reference values.

\section{Data sets}

The following data sets have been used to assess the above defined performance indicators.

The signal status and signal availability indicators are obtained using the consolidated navigation daily files generated by the IGS from a 300+ globally distributed network of sensor stations. These files include both F/NAV and I/NAV navigation messages transmitted by all the Galileo satellites over a day [9]. The files are available on the following day.

The ranging accuracy indicator is assessed with respect to the Center for Orbit Determination in Europe (CODE) final precise satellite and precise clock product series for the IGS-MGEX project [10]. The precise products are provided at 5-min rate over one full day. These products are published with a latency of 14 days.

All four Galileo satellite were marked healthy for operational use on 11 February 2019 around mid-day. Thus, the time interval considered in this study covers the first operational year of the four satellites, i.e. from 12 February 2019 to 12 February 2020.

\section{RESULTS AND DISCUSSIONS}

This section reports on the performance of the signals transmitted by the latest quartet of Galileo satellites in terms of three signal performance indicators: status, availability and ranging accuracy. In addition, we complement with an analysis of the on-board clock performance over the considered time interval.

\section{A. Signal-in-Space Status}

As mentioned in Section II, the signal-in-space status for a Galileo satellite is evaluated through three main data flags: data validity status (DVS), signal healthy status (SHS) and signal-in-space accuracy (SISA). We present below the individual performance of these three flags. 
TABLE IV

Data Validity Status (DVS) and Signal Health Status (SHS) Flags. [Unit: \%]

\begin{tabular}{lrrrrrr}
\hline Satellite & DVS=0 & \multicolumn{5}{c}{ SHS=0 } \\
PRN & E5a & E1 & E5b & E5a & E1 & E5b \\
\hline E13 & 100.00 & 100.00 & 100.00 & 100.0 & 100.0 & 100.0 \\
E15 & 99.97 & 99.97 & 99.97 & 100.0 & 100.0 & 100.0 \\
E33 & 98.42 & 98.42 & 98.42 & 97.9 & 97.9 & 97.9 \\
E36 & 100.00 & 100.00 & 100.00 & 100.0 & 100.0 & 100.0 \\
\hline
\end{tabular}

1) Data Validity Status: DVS flag transmitted on E5a, E1 and E5b is coded on 1 bit. This means that DVS values may be 0 (navigation data valid) or 1 (working without guarantee). [6]

Table IV summarises the data validity for all signal transmitted by the four Galileo satellites during the first operational year. One can notice that data transmission from E13 and E36 was valid for 100\% of the time, whereas for the other two satellites, the data validity accounted for 99.97 (E15) and 98.42 (E33) percent of the time, respectively. E15 transmitted working without guarantee data on 4 June 2019 for about three hours. E33 was one of the six satellites that transmitted working without guaranteed flagged data for 138 hours in connection to the Galileo technical incident in July 2019 [11].

2) Signal Health Status: SHS flag transmitted on E5a, E1 and E5b is coded on 2 bits. Thus, the flag can take four values: 0 (OK), 1 (out of service), 2 (will be out of service) or 3 (in testing) [6].

Table IV on the right hand side summarises the health flag for all signals transmitted by the four Galileo satellites during the first operational year. The transmission of a healthy signal was clean and clear for three of the four satellites (E13, E15, E36), whereas E33 was out of service for more than 180 hours in April 2019 as announced in NAGU 2019009.

3) Signal-in-Space Accuracy: SISA flag is a prediction at 1-sigma standard deviation of the quality of the transmitted signal. The flag can take values from 0 to 255 . The transmitted standard index is 107 that corresponds to a SISA value of $3.12 \mathrm{~m}$. If the prediction is not available, the transmitted index is 255 and corresponds to No Accurate Prediction Available (NAPA). NAPA is an indicator of a potential anomalous signal-in-space [6]. Please notice that SISA flag refers to the dual-frequency signal combinations.

Table V summarises the SISA transmission for E1/E5a and E1/E5b signals from all four satellites during the first operational year. All satellites transmitted standard SISA value (i.e. $3.12 \mathrm{~m}$ ) for more than $93 \%$ of the time for all signals, with E36 performing slightly better than the other satellites. In addition, SISA values higher than $3.12 \mathrm{~m}$ accounted for about $4 \%$ of the time. Maximum SISA value 
TABLE V

SISA FLAG AND NAPA EVENTS [UNIT: \%]

\begin{tabular}{|c|c|c|c|c|c|}
\hline \multirow[t]{2}{*}{ Signal } & \multicolumn{4}{|c|}{ Satellite PRN } & \multirow{2}{*}{$\begin{array}{l}\text { SISA } \\
\text { value }\end{array}$} \\
\hline & E13 & E15 & E33 & E36 & \\
\hline \multirow[t]{3}{*}{ E1/E5a } & 93.56 & 94.65 & 93.57 & 95.65 & $S I S A=3.12$ \\
\hline & 3.80 & 3.79 & 4.01 & 3.83 & $S I S A>3.12$ \\
\hline & 2.65 & 1.56 & 2.43 & 0.52 & $N A P A$ \\
\hline \multirow[t]{3}{*}{$\mathrm{E} 1 / \mathrm{E} 5 \mathrm{~b}$} & 93.79 & 94.08 & 93.99 & 94.04 & $S I S A=3.12$ \\
\hline & 3.80 & 3.79 & 4.01 & 3.80 & $S I S A>3.12$ \\
\hline & 2.41 & 2.13 & 2.01 & 2.16 & $N A P A$ \\
\hline
\end{tabular}

was $5.52 \mathrm{~m}$ and accounted for half the time (i.e. about $2 \%$ of the entire operational period). Furthermore, NAPA values for all signals were transmitted by all four satellites for more than $2 \%$ of the time, except E1/E5a signal from E15 for which NAPA values accounted for only $1.56 \%$ of the time. When NAPA value is transmitted in the navigation message, the signal-in-space status changes to marginal. Thus, the NAPA events are one of the main other reasons contributing to reduced signal-in-space availability.

\section{B. Signal-in-Space Availability}

The signal-in-space availability is mainly influenced by the data validity, signal health, NAPA events and age of navigation data.

Fig. 2 illustrates the per-satellite availability for all signals and satellites for the first operational year. The overall availability is greater than $95 \%$ for every single-frequency (E1, E5a, E5b) and dual-frequency combination (E1/E5a, E1/E5b). This performance is well above the $87 \%$ representing the minimum performance level (MPL) threshold specified in Section 3 of the Service Definition Document [5].

To further understand the results showed in Fig. 2, we broke down the one-year long availability into monthly values evaluated individually per frequency combination and satellite. These monthly availability values show that:

- $100 \%$ availability was achieved for at least 7 out of 12 months with a maximum of 9 months for E36.

- lower than 87\% availability was achieved for E33 in April 2019, all satellites in July 2019, and E36 in Sep 2019.

- the lowest availability (75\%) was encountered in April 2019 for E33. The satellite was out of service for more than a week due to a planned outage announced in NAGU 2019009. 


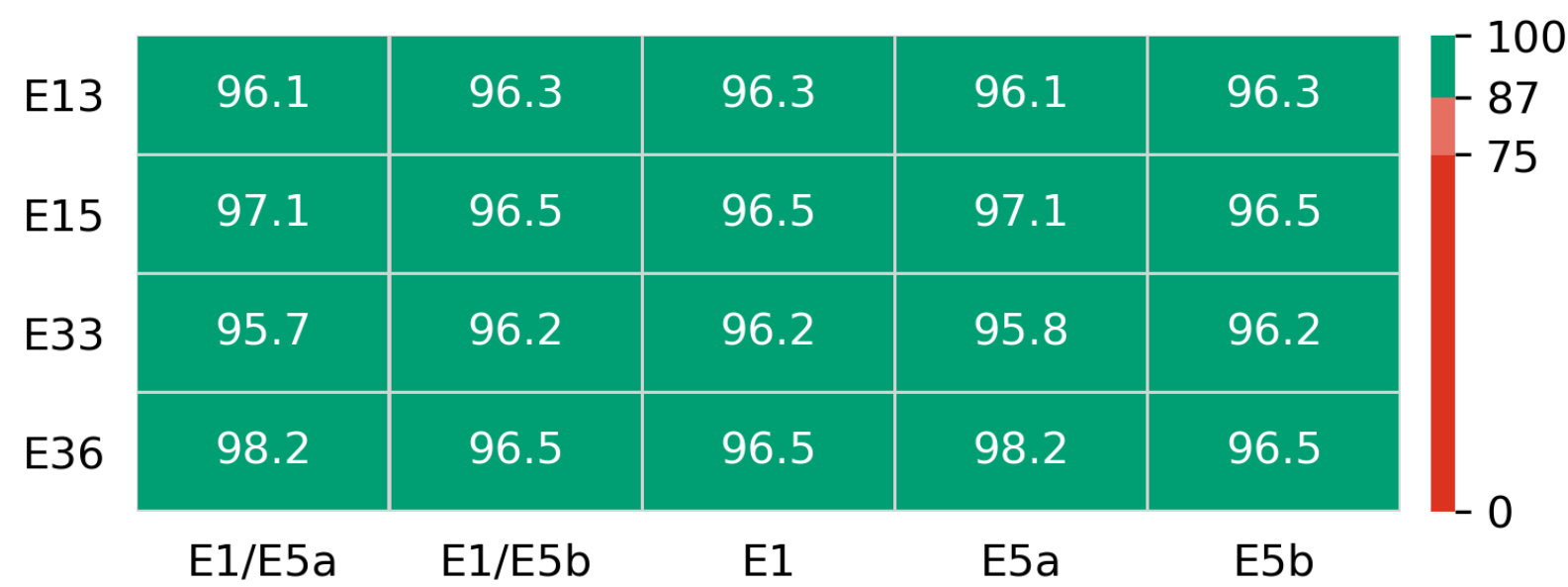

Fig. 2. Signal-in-Space availability per-satellite transmitting a healthy signal during the first operational year. [Unit: \%]

- around $80 \%$ availability was encountered for all satellites and signals in July 2019 due to the technical incident that affected the ground segment of the Galileo satellite system [11]. The incident meant that the navigation data was not refreshed for more than 120 hours.

- 83\% availability was achieved for E36 in September 2019 only for I/NAV-related signals (E1, E5b, $\mathrm{E} 1 / \mathrm{E} 5 \mathrm{~b})$ due to the transmission of the NAPA values.

\section{Signal-in-Space Ranging Accuracy}

Fig.3 depicts the signal-in-space ranging accuracy for the individual satellites and E1/E5a signal combination as the 95th percentile of the global average of the instantaneous SISE over the one-year operational period. The overall accuracy is in the range of 0.20 to $0.27 \mathrm{~m}$. This is well below the 7 $\mathrm{m}$ threshold specified as the minimum performance level [5] and in line with the findings for the other satellites in the Galileo constellation reported in [1]-[4].

Fig.4 shows the monthly values of the ranging accuracy for the individual satellites and E1/E5a signal combination. The $95 \%$ metric is used again. The monthly ranging accuracy varies from 0.17 to $0.33 \mathrm{~m}$. The best monthly metric is $0.17 \mathrm{~m}$. It was achieved for E15 and E36 in September and March 2019, respectively. The best daily metric is $0.10 \mathrm{~m}$. It was achieved on 30 November 2019 for E15 and 24 March 2019 for E36. Overall, E15 and E36 exhibit similar performance levels, 13\% better than E33 and $27 \%$ better than E13. The latter also exhibits the largest monthly variations. 


\section{5th percentile Global Average Signal in Space Error}

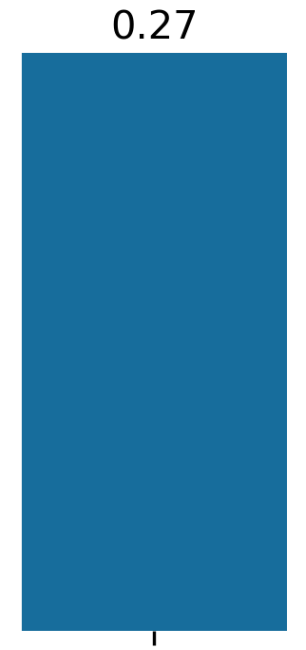

E13

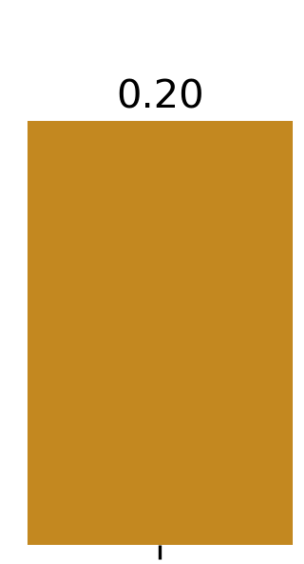

E15

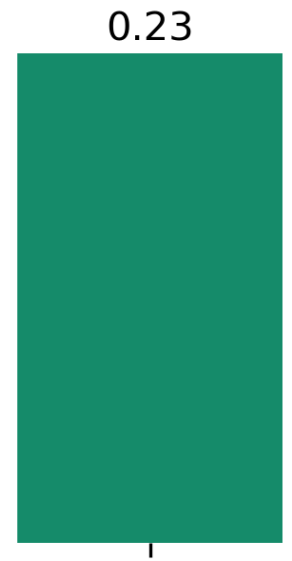

E33

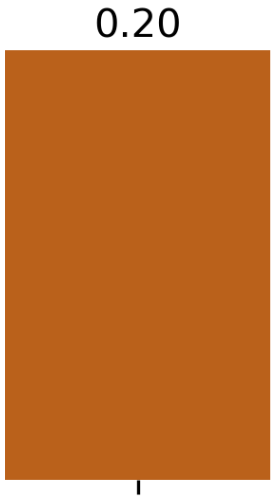

E36

Fig. 3. Ranging accuracy for the individual satellites and E1/E5a signal during the reporting period. [Unit: $\mathrm{m}$ ]

\section{On-board satellite clocks}

Atomic clock are the heart of any satellite-base navigation system. Galileo satellites are equipped with four atomic clocks: two passive hydrogen maser (PHM) and two Rubidium Atomic Frequency Standard (RAFS) clocks. Thus, it is also interesting to look at how the on-board satellite clocks have performed over the first operational year. It should be mentioned that all four satellites work on PHM clock.

Fig. 5 illustrates the first-order difference (i.e. current minus previous) of the satellite clock biases as derived from the precise products at 5-minute rate. In addition, the daily median difference is displayed by the dark line. Furthermore, the overall mean and the corresponding standard deviation of these differences are given in the top right corner.

The overall short-term performance is very good with first-order differences at the picosecond level. The differences for E13, E15 and E36 exhibit pick-to-pick variations below 1 ps/s and standard deviations around $0.1 \mathrm{ps} / \mathrm{s}\left(10^{-13} \mathrm{~s} / \mathrm{s}\right)$, whereas for E33 the differences showed less than $2 \mathrm{ps}$ range and $0.2 \mathrm{ps} / \mathrm{s}$ standard deviation. Additionally, E33 plot reveals us two interesting things. First, there is a clear jump in the beginning of April. This is associated with the planned outage and maintenance work mentioned in the previous sections. The maintenance work also meant the switch to another on-board satellite clock. This is the main reason of the jump. Second, an unusual, U-shape variation took place during September-November 2019. Initially, the satellite clock biases exhibits rapid negative drift in the first 


\begin{tabular}{|c|c|c|c|c|}
\hline Feb-19- & 0.30 & 0.20 & 0.26 & 0.20 \\
\hline Mar-19 - & 0.27 & 0.19 & 0.23 & 0.17 \\
\hline Apr-19- & 0.25 & 0.22 & 0.22 & 0.20 \\
\hline May-19 - & 0.25 & 0.21 & 0.22 & 0.21 \\
\hline Jun-19 - & 0.24 & 0.20 & 0.24 & 0.19 \\
\hline Jul-19 - & 0.26 & 0.20 & 0.22 & 0.20 \\
\hline Aug-19- & 0.24 & 0.19 & 0.20 & 0.23 \\
\hline Sep-19 - & 0.25 & 0.17 & 0.21 & 0.19 \\
\hline Oct-19- & 0.28 & 0.19 & 0.24 & 0.21 \\
\hline Nov-19- & 0.33 & 0.18 & 0.22 & 0.21 \\
\hline Dec-19- & 0.29 & 0.21 & 0.24 & 0.20 \\
\hline Jan-20 - & 0.29 & 0.20 & 0.24 & 0.19 \\
\hline Feb-20 - & 0.30 & 0.19 & 0.21 & 0.19 \\
\hline & E13 & E15 & E33 & E36 \\
\hline
\end{tabular}

Fig. 4. 95th percentile of the monthly global average SISE for E1/E5a signal during the reporting period. [Unit: m]

half of September, then the drift shows a plateauing behaviour until the end of October, followed by a rapid positive drift until mid of November. Afterwards, the positive drift stabilises. Moreover, one can also notice a rapid positive drift for E15 in the beginning of November, whereas a milder effect may also 
be seen in E13. It is rather challenging to interpret these behaviour changes at this point. There might be controlled or side-effects of potential adjustments in the overall constellation carried out by the ground control segment. Further investigations are needed to thoroughly explain these findings.

\section{SUMmARY AND CONCLUSIONS}

Galileo constellation increased to 22 operational satellites on 11 February 2019, when the latest quartet of Galileo satellites were marked available for operation. The quartet included GSAT0219 (E36), GSAT0220 (E13), GSAT0221 (E15) and GSAT0222 (E33). A closer look at the individual performance of these four Galileo satellites has been conducted for the first time. The performance covers three signalin-space indicators: status, availability and accuracy. In addition, we also analysed the performance of the on-board satellite clock by looking at the precise satellite clock biases. The main take away points are as follows.

1) The data validity and signal health status were $100 \%$ for three of the satellites (E13, E15, and E36). The fourth satellite (E33) achieved lower percentage mainly due to the planned outage in April 2019.

2) The overall signal-in-space availability was higher than $95 \%$ for all signals and satellites. Individually, the satellites achieved $100 \%$ availability for seven to nine months of the year. For the other months, the availability was reduced due to the Galileo technical incident in July 2019 (all satellites), signal outage (E33 in April 2019 and E36 in September 2019), or transmission of NAPA values that changes the status of the signal-in-space from healthy to marginal. Overall, NAPA events accounted for about $2 \%$ of the time..

3) The signal-in-space ranging accuracy annual value expressed as 95th global average SISE was found to be $0.20 \mathrm{~m}$ (E15 and E36), $0.23 \mathrm{~m}$ (E33) and $0.27 \mathrm{~m}$ (E13). The monthly values varied from 0.17 to $0.33 \mathrm{~m}$.

4) The on-board satellite clock performance has been very high in the first operational year. The clocks revealed picosecond level variations with about $10^{-13} \mathrm{~s} / \mathrm{s}$ standard deviations. In addition, E33 experienced a clock switch in April 2019 and unusual variations in the drift rate from September to November 2019.

Our findings indicate a robust performance and high signal reliability for the youngest Galileo satellites in the constellation. The satellites increase the world-wide availability of Galileo satellites. Thus, they contribute to the Galileo's global popularity in the satellite-based positioning and navigation user community. 


\section{ACKNOWLEDGEMENT}

The research study was carried out as part of the 4020-1120G project funded by the National Land Survey of Finland. SISE was computed using in-house software developed at Chalmers. We acknowledge the European Union and the European GNSS Agency (GSA) for supporting the cooperation of the Galileo Reference Center (GRC) [12] with Member States within the GRC FiNLS project, and co-financed (Grant agreement nr. GSA/GRANT/04/2016), in support of an independent monitoring of the Galileo system performance.

\section{REFERENCES}

[1] P. Steigenberger and O. Montenbruck, "Galileo status: orbits, clocks, and positioning," GPS Solutions, vol. 21, no. 2, pp. 319-331, Apr. 2017. [Online]. Available: https://doi.org/10.1007/s10291-016-0566-5

[2] G. Galluzzo, R. L. Rodriguez, R. Morgan-Owen, S. Binda, D. Blonski, P. Crosta, F. Gonzalez, J. M. Garcia, X. Otero, N. Sirikan, M. Spangenberg, E. Spinelli, R. Swinden, and S. Wallner, "Galileo System Status, Performance Metrics and Results," in Proceedings of the 2018 International Technical Meeting of The Institute of Navigation, Reston, Virginia, jan 2018, pp. 790-809.

[3] O. Montenbruck, P. Steigenberger, and A. Hauschild, "Multi-GNSS signal-in-space range error assessment Methodology and results," Advances in Space Research, vol. 61, no. 12, pp. 3020 - 3038, 2018. [Online]. Available: http://www.sciencedirect.com/science/article/pii/S0273117718302813

[4] W. Wu, F. Guo, and J. Zheng, "Analysis of Galileo signal-in-space range error and positioning performance during 2015-2018," Satellite Navigation, vol. 20, p. 6, Feb. 2020. [Online]. Available: https://doi.org/10.1186/s43020-019-0005-1

[5] European GNSS (Galileo), “Open Service - Service Definition Document," May 2019, issue 1.1. [Online]. Available: https://www.gsc-europa.eu/sites/default/files/sites/all/files/Galileo-OS-SDD_v1.1.pdf

[6] European GNSS (Galileo) Open Service, "Signal-in-Space Interface Control Document," Dec. 2016, issue 1.3. [Online]. Available: https://www.gsc-europa.eu/sites/default/files/sites/all/files/Galileo-OS-SIS-ICD.pdf

[7] _ _ "Ionospheric Correction Algorithm for Galileo Single Frequency Users," Sep. 2016, issue 1.2. [Online]. Available: https://www.gsc-europa.eu/sites/default/files/sites/all/files/Galileo_Ionospheric_Model.pdf

[8] M. Falcone, J. Hahn, and T. Burger, Galileo. Cham: Springer International Publishing, 2017, pp. 247-272. [Online]. Available: https://doi.org/10.1007/978-3-319-42928-1_9

[9] G. Johnston, A. Riddell, and G. Hausler, The International GNSS Service. Cham: Springer International Publishing, 2017, pp. 967-982. [Online]. Available: https://doi.org/10.1007/978-3-319-42928-1_33

[10] D. Prange, Lars ahd Arnold, R. Dach, S. Schaer, D. Sidorov, P. Stebler, A. Villiger, and A. Jäggi, "Code product series for the igs mgex project," 2018, issue 1.1. [Online]. Available: http://www.aiub.unibe.ch/download/CODE_MGEX/

[11] Maanmittauslaitos.fi. (2019, Jul.) Galileo incident of July 11th to 17th. [Online]. Available: https://www.maanmittauslaitos. fi/en/topical_issues/galileo-incident-july-11th-17th

[12] P. Buist, M. Porretta, A. Mozo, and H. Tork, "The Galileo Reference Centre and Its Role in the Galileo Service Provision," in 69th International Astronautical Congress (IAC), Bremen, Germany, Oct. 1-5, 2018, p. 11. 

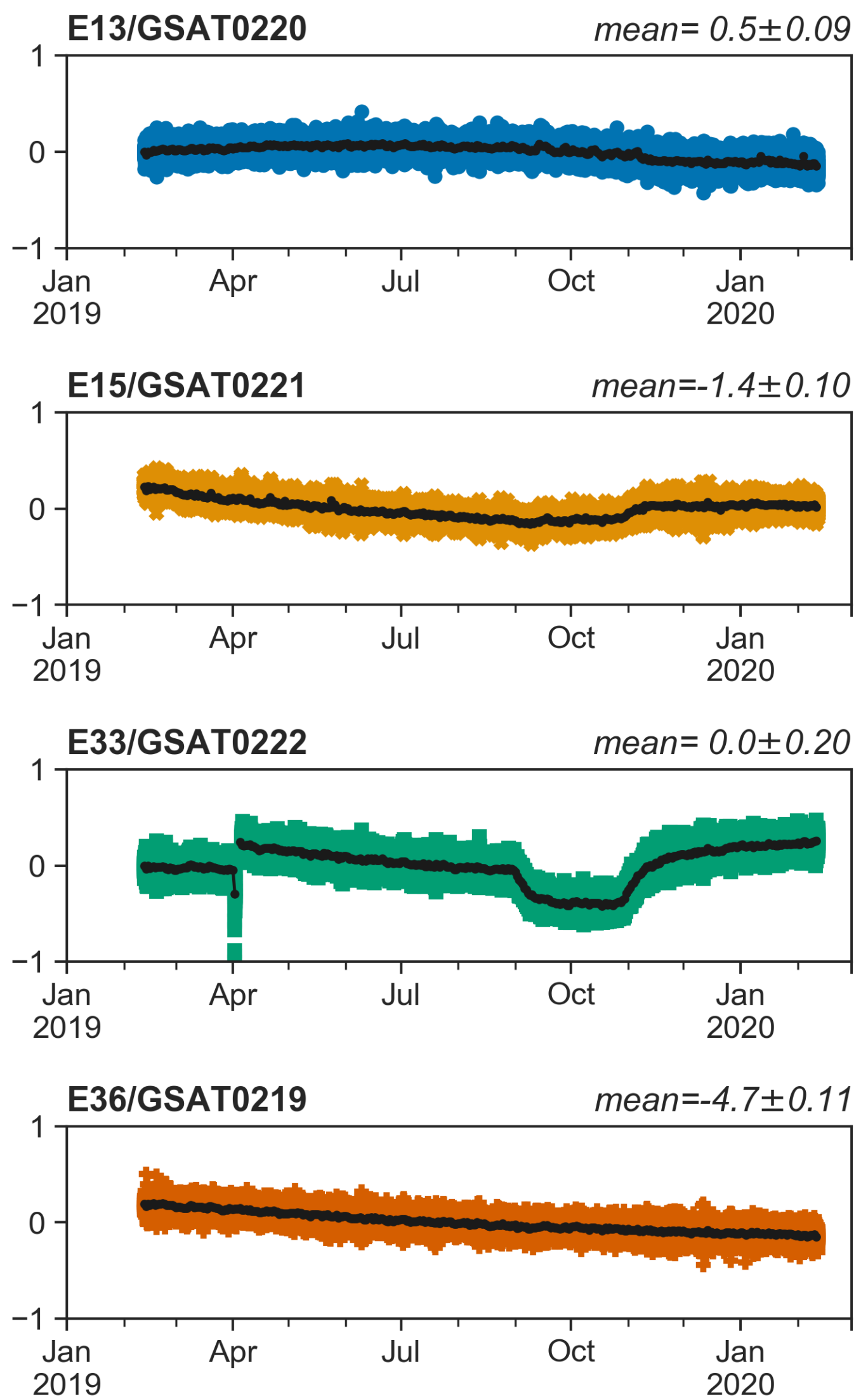

Fig. 5. First-order differences (i.e., current minus previous) derived from the satellite clock biases at 5-min rate (coloured dots) the daily mean difference (dark line). The plotted data has been centred around the overall mean. [Unit: p8RAFT 